Heine-Jahrbuch 
Herausgegeben in Verbindung mit der Heinrich-Heine-Gesellschaft 


\title{
HEINE-JAHRBUCH 2003
}

\author{
42. Jahrgang \\ Herausgegeben von Joseph A. Kruse \\ Heinrich-Heine-Institut \\ der Landeshauptstadt Düsseldorf
}

Verlag J. B. Metzler

Stuttgart . Weimar 


\title{
Anschrift des Herausgebers: Joseph A. Kruse \\ Heinrich-Heine-Institut
}

Bilker Straße I2-I4, 40213 Düsseldorf

Redaktion: Karin Füllner und Marianne Tilch

Bibliografische Information Der Deutschen Bibliothek

Die Deutsche Bibliothek verzeichnet diese Publikation in der Deutschen Nationalbibliografie; detaillierte bibliografische Daten sind im Internet über <http://dnb.ddb.de> abrufbar.

\author{
ISBN 978-3-476-o1979-o \\ ISBN $978-3-476-02887-7$ (eBook) \\ DOI 10.1007/978-3-476-02887-7 \\ ISSN $0073^{-1692}$
}

Dieses Werk einschließlich aller seiner Teile ist urheberrechtlich geschützt. Jede Verwertung außerhalb der engen Grenzen des Urheberrechtsgesetzes ist ohne Zustimmung des Verlages unzulässig und strafbar. Das gilt insbesondere für Vervielfältigungen, Übersetzungen, Mikroverfilmungen und die Einspeicherung und Verarbeitung in elektronischen Systemen.

(C) 2003 Springer-Verlag GmbH Deutschland

Ursprünglich erschienen bei J. B. Metzlersche Verlagsbuchhandlung und Carl Ernst Poeschel Verlag GmbH in Stuttgart 2003

www.metzlerverlag.de

Info@metzlerverlag.de 


\section{Inhalt}

Siglen

IX

Aufsätze

I.

"Die dritte Säule meines lyrischen Ruhmes" - Heines "Romanzero".

Beiträge des Kolloquiums im Heinrich-Heine-Institut am 13. April 2002. Teil I

Ortwin Lämke und Christian Liedtke · Einleitung

Andreas Böhn · Erinnerungswelten. Geschichte und Exotik im "Romanzero" . . . . . . . . . . . . . . . . . . . . 3

Olaf Briese - Exil auf Erden. Facetten einer Zumutung in Heines

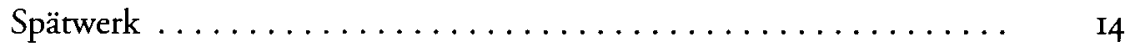

Jutta Nickel - Grabgeschichten. Zur Besichtigung einer Ortschaft im "Romanzero" ............................ 37

Robert Steegers • »Indezent und degoutant zugleich«. Intertextuelles in Heines "Romanzero" - am Beispiel August von Platen ........ 59

II.

Karin Sousa . Wahrheit und Widersprüche in Heinrich Heines $"$ Buch der Lieder" . . . . . . . . . . . . . . . . . . . .

Hilaria Gössmann · Heinrich Heines »Harzreise« und »Die Tänzerin von Izu« von Kawabata Yasunari. Eine literarische Gegenüberstellung .... 
Jörg Bernig • Vergessenheit und Instrumentalisierung. Die deutsche Heine-Rezeption im ersten Nachkriegsjahrzehnt ............ IO

Wolfgang Büttner - Der andere Herwegh - über sein Verhältnis zur internationalen Arbeiterbewegung..$\ldots \ldots \ldots \ldots \ldots \ldots$

\section{Kleinere Beiträge}

Sikander Singh - "Schickt einen Philosophen nach London; bey Leibe keinen Poeten!« - Heinrich Heine und Georg Christoph Lichtenberg . . I40

Gislinde Seybert . Heines "Loreley« und Puschkins »Sing mir nicht, schöne Frau«. Dekonstruktiv-linguistische Analyse von zwei Gedichten der Romantik ................. I50

Gerhart Söhn • Das verleugnete Heine-Porträt. Eine Dokumentation ... $\quad{ }_{158}$ Axel Seibert • Die Heine-Bank im Bremer Bürgerpark ........... $\quad{ }_{164}$

Heinrich-Heine-Institut. Sammlungen und Bestände. Aus der Arbeit des Hauses

Bernd Füllner und Christian Liedtke · Volltext, Web und Hyperlinks.

Das Heinrich-Heine-Portal und die digitale Heine-Edition ....... $\quad{ }_{17} 8$ Karin Füllner - "und gerade Heine überzeugt mich".

Das Düsseldorfer Studierenden-Kolloquium 2002 mit neuen Arbeiten über Heinrich Heine . . . . . . . . . . . . . . . . . . . . . . $\quad$ I88

Reden

Reden zur Verleihung des Heine-Preises 2002

Elfriede Jelinek . Österreich. Ein deutsches Märchen .......... 192

Stefanie Carp · Laudatio auf Elfriede Jelinek ............. $\quad 208$

Reden zur Verleihung der Ehrengabe der Heinrich-Heine-Gesellschaft 2003 Ingrid Bachér · Grußwort $\ldots \ldots \ldots \ldots \ldots \ldots \ldots \ldots \ldots \ldots \ldots \ldots \ldots \ldots \ldots$

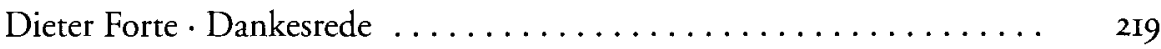

Manfred Durzak • Dieter Fortes Weg in die Literatur. Eine Laudatio .... 225 
Nachruf

Joseph A. Kruse · Erinnerung an Gerd Högener . . . . . . . . . . . 234

\section{Buchbesprechungen}

Ingrid Bodsch (Hrsg.) • "An den Rhein, an den Rhein ...«. Das malerische und romantische Rheinland in Dokumenten, Literatur und Musik

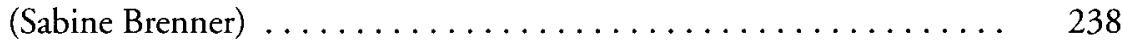

Reinhard Blänkner/Gerhard Göhler/Norbert Wasek (Hrsg.) · Eduard Gans (1797-1839). Politischer Professor zwischen Restauration und Vormärz (Volkmar Hansen) . . . . . . . . . . . . . . . . .

Roger F. Cook (Hrsg.) - A Companion to the Works of Heinrich Heine

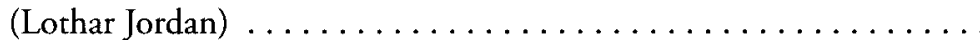

Enid und Bernhard Gajek (Hrsg.) • "Die Leidenschaft ist der Schlüssel zur Welt«. Der Briefwechsel zwischen Bettine von Arnim und Hermann von Pückler-Muskau 1832-1844 (Wolfgang Bunzel) . . . .

Hans Hörling (Hrsg.) - Die französische Heine-Kritik. Band 3.

Rezensionen und Notizen zu Heines Werken aus den Jahren 1846-1856 (Bernd Füllner) . . . . . . . . . . . . . . . . . . 245

Gerhart Hoffmeister . Heine in der Romania (Joseph A. Kruse) . . . . . 247

Hans Kaufmann . Der Januskopf des Utopischen. Texte, Gespräche, Erinnerungen (Joseph A. Kruse) . . . . . . . . . . . . . . . . . . 249

Bernd Kortländer · Heinrich Heine (Sikander Singh) . . . . . . . . . 250

Mario Kramp · Heinrich Heines Kölner Dom. Die »armen Schelme vom Domverein« im Pariser Exil 1842-1848 (Sabine Brenner) . . . . .

Michael Perraudin - Literatur, the Volk and the Revolution in Mid-Nineteenth Century Germany (Bernd Kortländer)

Adam Smykowski · Heinrich Heines ,Lyrisches Intermezzo in Vetonungen von Robert Schumann und Robert Franz/Beate Julia Perrey - Schumann's 'Dichterlieber and Early Romantic Poetics. Fragmentation of Desire (Thomas Synofzik) . . . . . . . . . . . . . . . . . 254

Jürgen Wilhelm/Frank Günter Zehnder - Der Rhein. Bilder und Ansichten von Mainz bis Nijmwegen (Sabine Brenner) . . . . . . . .

Heine-Literatur 2002/2003 mit Nachträgen . . . . . . . . . . . . 
Veranstaltungen des Heinrich-Heine-Instituts und der Heinrich-Heine-Gesellschaft e. V. Januar bis Dezember 2002 .................... 279

Ankïndigung des Düsseldorfer Studierenden-Kolloquiums $2004 \ldots \ldots \ldots \quad 288$

Abbildungen ............................. 289

Hinweise für die Autoren . . . . . . . . . . . . . . . . . . . 290

Mitarbeiter des Heine-Jahrbuchs $2003 \ldots \ldots \ldots \ldots \ldots \ldots \ldots \ldots \ldots . . \ldots 292$ 


\section{Siglen}

\section{H. Heine: Werke und Briefe}

B

DHA

HSA
$=$ Heinrich Heine: Sämtliche Schriften. Hrsg. von Klaus Briegleb. München: Hanser 1968-1976, 6 Bände (6, II = Register)

$=$ Heinrich Heine: Historisch-kritische Gesamtausgabe der Werke. In Verbindung mit dem Heinrich-Heine-Institut hrsg. von Manfred Windfuhr. Hamburg: Hoffmann und Campe 1973-1997, I6 Bände

= Heinrich Heine: Werke, Briefwechsel, Lebenszeugnisse. Säkularausgabe. Hrsg. von den Nationalen Forschungs- und Gedenkstätten der klassischen deutschen Literatur in Weimar (seit 1991: Stiftung Weimarer Klassik) und dem Centre National de la Recherche Scientifique in Paris. Berlin und Paris: Akademie und Editions du CNRS $1970 \mathrm{ff}$.

2. Weitere Abkürzungen

Galley/Estermann = Eberhard Galley und Alfred Estermann (Hrsg.): Heinrich Heines Werk im Urteil seiner Zeitgenossen. Hamburg: Hoffmann und Campe 1981-1992, 6 Bände.

auf der Horst/Singh $=$ Christoph auf der Horst und Sikander Singh (Hrsg.): Heinrich Heine im Urteil seiner Zeitgenossen. Begründet von Eberhard Galley und Alfred Estermann. Stuttgart/Weimar: Metzler $2002 \mathrm{ff}$.

HJb = Heine-Jahrbuch. Hrsg. vom Heinrich-Heine-Institut Düsseldorf. Hamburg: Hoffmann und Campe 1962-r994; Stuttgart: Metzler 1995 ff.

Mende $\quad$ = Fritz Mende: Heinrich Heine. Chronik seines Lebens und Werkes. Berlin: Akademie ${ }^{1} 1970 ;{ }^{2} 198 \mathrm{r}$

Seifert = Siegfried Seifert: Heine-Bibliographie 1954-1964. Berlin und Weimar: Aufbau 1968

Seifert/Volgina = Siegfried Seifert und Albina A. Volgina: Heine-Bibliographie 1965-1982. Berlin und Weimar (Hrsg.): Begegnungen mit Heine. Berichte der Zeitgenossen. Hamburg: Hoffmann und Campe 1973, 2 Bände

Wilamowitz = Erdmann von Wilamowitz-Moellendorf und Günther Mühlpfordt $(\dagger)$ : Heine-Bibliographie 1983-1995. Stuttgart und Weimar: Metzler 1998

Wilhelm/Galley = Gottfried Wilhelm und Eberhard Galley: Heine-Bibliographie [bis 1953]. Weimar: Arion 1960, 2 Bände 